\title{
Preface to the Special Issue on Genetic Improvement
}

\author{
Justyna Petke ${ }^{1}$
}

Published online: 27 August 2016

(C) Springer Science+Business Media New York 2016

I am delighted to present this special issue of the Genetic Programming and Evolvable Machines journal on Genetic Improvement, which has emerged as a new separate field of research only in the last few years. Genetic improvement applies evolutionary and search-based optimisation methods to the improvement of existing software. It could be used, for instance, to automate the process of software repair or to minimise bandwidth, memory or energy consumption.

Genetic programming can use human-written software as a feed stock for genetic improvement and is able to evolve mutant software tailored to solving particular problems. Other interesting areas are automated software transplantation, as well as "grow-and-graft" genetic programming, where software is incubated outside its target human written code and subsequently grafted into it via genetic improvement.

Work on software repair, specialisation and transplantation resulted in four "Humies", awarded for human-competitive results produced by genetic and evolutionary computation (http://www.human-competitive.org/awards). Genetic improvement work has also won multiple best papers awards.

The success and rapid growth of this emergent area led to a dedicated event series, Genetic Improvement Workshops (http://www.geneticimprovementofsoftware.com), started in 2015 and organised in conjunction with the Genetic and Evolutionary Computation Conference (GECCO). It is thus also the ripe time for a special journal issue on genetic improvement, the purpose of which is to investigate the work and trends in this new field of research. Following an open call for papers, the three articles that comprise this special issue present the latest achievements in the field, one of which was handled by the editor-in-chief to avoid conflict of interest.

In "Genetic Improvement of GPU Software" William B. Langdon et al. present a collection of several successful applications of genetic improvement. These range

Justyna Petke

j.petke@ucl.ac.uk

1 Department of Computer Science, University College London (UCL), London, UK 
from upgrading legacy code for new hardware through software efficiency improvement to automated software parallelisation.

In "Trading between Quality and Non-functional Properties of Median Filter in Embedded Systems" Zdenek Vasicek and Vojtech Mrazek investigate the possibilities and opportunities for improving non-functional properties of software, such as execution time, code size and energy consumption. They focus on applying genetic improvement techniques to the median functions implemented using comparator networks.

Finally, in "Online Genetic Improvement on the JVM with ECSELR" Kwaku Yeboah-Antwi and Benoit Baudry present an online genetic improvement framework called ECSELR. It has the ability to improve and adapt inside a target software system enabling it to evolve and adapt at runtime without any external dependencies or human intervention.

Collectively, these three papers illustrate the diverse range of applications of genetic improvement and provide a detailed compilation of the various issues currently being investigated in this new research area.

I would like to thank the editor-in-chief, the authors and the reviewers for contributing to this special issue on genetic improvement. It will undoubtedly encourage engaging work in this exciting field. I hope you take some inspiration from this special issue for your own future research. Enjoy reading! 